Correspondence

John E. Moore

jemoore@niphl.dnet.co.uk

Received 24 January 2011

Accepted 26 April 2011

\section{Identification and characterization of breakthrough contaminants associated with the conventional isolation of Mycobacterium tuberculosis}

\author{
Michaela McClean, ${ }^{1,2}$ Timothy Stanley, ${ }^{1}$ Sharon Stanley, ${ }^{1}$ \\ Yasunori Maeda, ${ }^{2,3}$ Colin E. Goldsmith, ${ }^{3}$ Richard Shepherd, ${ }^{4}$ \\ B. Cherie Millar, ${ }^{3}$ James S. G. Dooley ${ }^{2}$ and John E. Moore ${ }^{2,3}$
}

${ }^{1}$ Northern Ireland Mycobacterium Reference Laboratory, Department of Medical Microbiology, Royal Group of Hospitals, Grosvenor Road, Belfast, Northern Ireland, UK

${ }^{2}$ School of Biomedical Sciences, University of Ulster, Cromore Road, Coleraine, Northern Ireland BT52 1SA, UK

${ }^{3}$ Northern Ireland Public Health Laboratory, Department of Bacteriology, Belfast City Hospital, Lisburn Road, Belfast, Northern Ireland BT9 7AD, UK

${ }^{4}$ Regional Respiratory Centre, Department of Respiratory Medicine, Belfast City Hospital, Lisburn Road, Belfast, Northern Ireland BT9 7AB, UK

Breakthrough contamination of tuberculosis (TB) cultures is a problem in that it allows the overgrowth of another bacterium present in the sputum specimen, which can potentially mask the presence of Mycobacterium tuberculosis. The aim of this study was to isolate and characterize the bacterial organisms responsible for such overgrowth and contamination, and to examine their susceptibility to (i) various chemical selective decontamination steps and (ii) antibiotics in liquid culture media, in an attempt to develop a method to help alleviate contamination problems associated with the conventional isolation of $M$. tuberculosis from routine patient sputum specimens. Bacterial contaminants from 102 routine sputum cultures were identified molecularly by $16 \mathrm{~S}$ rRNA gene PCR and direct sequencing from contaminated Löwenstein-Jensen (LJ) slopes and BacT/Alert liquid medium. It was found that the contaminants from $L J$ slopes belonged to 11 different genera and were composed largely of Gram-negative organisms (84.9\%; 45/53), whereas the liquid culture contaminants belonged to 13 different genera, with $37 / 66$ isolates (56.1\%) being Gram-negative. Pseudomonas aeruginosa was the dominant contaminant in both media. The effect of six different selective decontamination protocols was examined. Four of the six methods were effective at eliminating all culturable organisms present; these were $5 \%$ oxalic acid, $5 \%$ oxalic acid/2\% NaOH, $5 \%$ oxalic acid/4\% NaOH and $1 \%$ chlorhexidine. $\mathrm{NaOH}$ at a concentration of 2 or $4 \%$ was less effective as it was unable to eliminate all organisms of each species tested, with the exception of $P$. aeruginosa. In conclusion, breakthrough contamination of TB cultures is due to a diverse range of at least 17 different bacterial genera, with $P$. aeruginosa and Staphylococcus epidermidis accounting for the dominant contaminating flora. Employment of chemical decontaminating protocols solely involving $\mathrm{NaOH}$ may lead to higher rates of contamination. Where such contamination is encountered, TB laboratories should consider the reprocessing of such sputum samples with an alternative decontamination method such as $1 \%$ chlorhexidine.
Abbreviations: CF, cystic fibrosis; LJ, Löwenstein-Jensen; NALC, $\mathrm{N}$-acetyl-L-cysteine; NIMRL, Northern Ireland Mycobacterium Reference Laboratory; TB, tuberculosis.

The GenBank/EMBL/DDBJ accession numbers for the partial $16 \mathrm{~S}$ rRNA gene locus sequences for each of the species identified in this study are FJ232593-FJ232619.

\section{INTRODUCTION}

The Northern Ireland Mycobacterium Reference Laboratory (NIMRL), Department of Bacteriology, Royal Victoria Hospital, Belfast, is the regional reference laboratory for tuberculosis (TB) in Northern Ireland. Presently, it processes approximately 7500 clinical specimens per year and receives isolates of clinical Mycobacterium species (including 
Mycobacterium tuberculosis, as well as atypical mycobacteria) from other hospital laboratories within Northern Ireland for identification, antibiotic susceptibility testing and epidemiological characterization. Over the last decade, the NIMRL has noted a local increase in contamination rates with breakthrough contaminants, as shown in Fig. 1, i.e. the overgrowth of primary cultures by antibiotic-resistant nonmycobacterial contaminating organisms, which compromise the ability to detect $M$. tuberculosis due to competition effects and physical masking of TB organisms, which have relatively slow growth properties. This contamination rate was generally $5 \%$ but increased steadily to $10 \%$ between 2000 and 2005 (Fig. 1). The downstream effect of such overgrowth and contamination potentially compromises the ability to detect and isolate M. tuberculosis, which may result in the non-identification of $M$. tuberculosis in sputum and no isolation of an organism on which to perform antibiotic susceptibility testing for patient management and infection control, as well as preventing the detection of multidrugresistant TB.

Therefore, it was the aim of this study to isolate and characterize the bacterial organisms responsible for such overgrowth and contamination, in terms of (i) their susceptibility to various chemical selective decontamination steps and (ii) their antibiotic resistance in liquid culture media, in an attempt to develop a method to help alleviate contamination problems associated with the conventional isolation of $M$. tuberculosis from routine patient sputum specimens.

\section{METHODS}

Bacterial contaminants. Routine clinical sputum specimens that had been sent to the NIMRL for TB workup by the conventional cultural isolation/examination of $M$. tuberculosis were obtained from patients $(n=102)$ during the period 2007-2008. All sputum specimens were processed routinely for the conventional detection of M. tuberculosis using a $4 \%(\mathrm{w} / \mathrm{v}) \mathrm{NaOH}$ decontamination step, which involved extended culture on Löwenstein-Jensen (LJ) agar, as well as in liquid culture medium, using the automated MB/BacT (BacT/Alert MP; bioMérieux) liquid culture medium. Consecutively contaminated
LJ slopes and BacT/Alert liquid medium were identified and set aside for further processing as part of this study. In total, 102 sputum specimens were identified as being contaminated, including unpaired contaminated LJ slopes $(n=41)$ and contaminated BacT/Alert medium $(n=50)$, as well as 11 paired contaminated LJ slopes plus contaminated BacT/Alert medium. Bacterial contaminants were isolated by subculture of LJ slopes and BacT/Alert medium onto Columbia blood agar (Oxoid), supplemented with $5 \%(\mathrm{v} / \mathrm{v})$ defibrinated horse blood for $24-48 \mathrm{~h}$ at $37{ }^{\circ} \mathrm{C}$ under aerobic conditions.

Viable culturable bacterial isolates $(n=119)$ were examined in order to obtain identification by $16 \mathrm{~S}$ rRNA gene PCR and direct automated sequencing, in accordance with a standard protocol, already established and published previously by our group (Xu et al., 2008).

Effect of different chemical selective decontamination protocols. Six different chemical selective decontamination methods were compared, which, together with other methods, have been described previously, as detailed in Table 1: (i) $2 \% \mathrm{NaOH}$, (ii) $4 \%$ $\mathrm{NaOH}$, (iii) $5 \%$ oxalic acid, (iv) $5 \%$ oxalic acid/2\% NaOH, (v) $5 \%$ oxalic acid $/ 4 \% \mathrm{NaOH}$ and (vi) $1 \%$ chlorhexidine. Seven bacterial contaminants were selected comprising the most frequently isolated contaminating organisms from the LJ slopes, as well as from the BacT/Alert liquid culture medium. These consisted of four wild-type Gram-negative organisms (Klebsiella pneumoniae, Pseudomonas aeruginosa, Serratia marcescens and Stenotrophomonas maltophilia) and three wild-type Gram-positive organisms (Enterococcus faecalis, Staphylococcus aureus and Staphylococcus epidermidis). Overnight cultures of each organism were inoculated individually into fresh sterile PBS to give a final cell density of approximately $6-7 \log _{10}$ c.f.u. $\mathrm{ml}^{-1}$. Equal volumes of inoculant and chemical decontaminant were used for each method ( $7 \mathrm{ml}$ each). The 2 and $4 \% \mathrm{NaOH}$ solutions were left to stand for $25 \mathrm{~min}$ after addition of the contaminant and

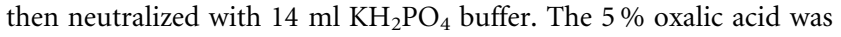

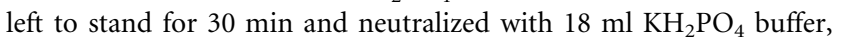
and the $1 \%$ chlorhexidine was left to stand for $15 \mathrm{~min}$ and the samples then washed with PBS (Ferroni et al., 2006). After incubation, the remaining culturable cells were enumerated using a spread plate technique and the counts were expressed as c.f.u. $\mathrm{ml}^{-1}$.

Determination of antibiotic susceptibility. Antibiotic susceptibility testing was performed using standard disc susceptibility testing techniques on bacterial contaminants isolated from the $\mathrm{LJ}$ slopes and the BacT/Alert liquid medium to determine how resistant the contaminants were to the antibiotics used as selective agents in the BacT/Alert liquid medium. The following discs were used: vancomycin $(30 \mu \mathrm{g})$, nalidixic acid $(30 \mu \mathrm{g})$, trimethoprim $(5 \mu \mathrm{g})$ and

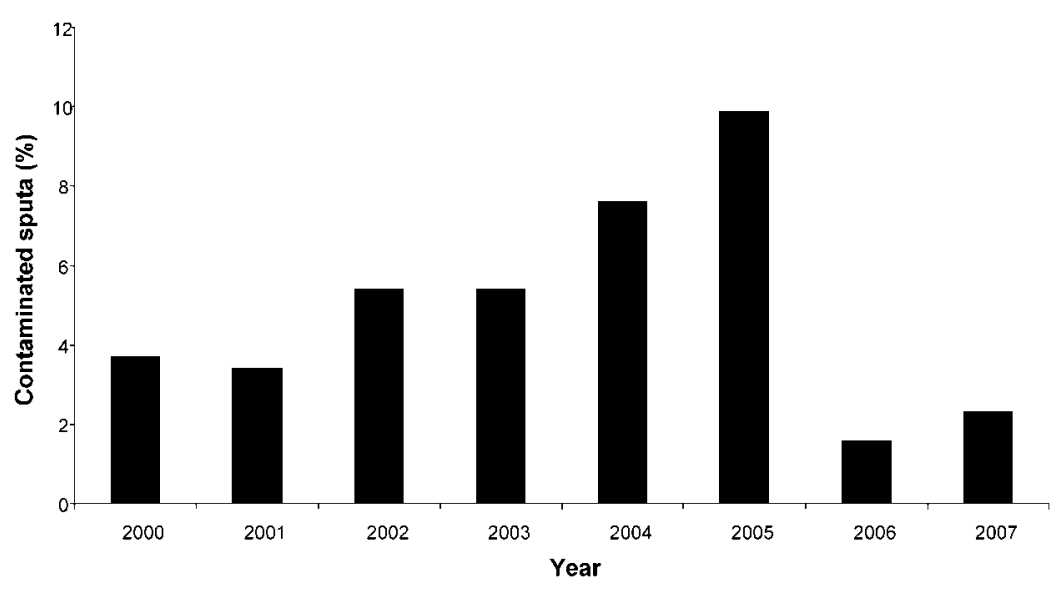

Fig. 1. Graph showing the annual occurrence of contaminated sputa for conventional TB workup at the NIMRL during the period 2000-2007. 
Table 1. Review of different chemical decontamination protocols used to aid in the laboratory diagnosis of M. tuberculosis

All percentages are w/v. TSP, trisodium phosphate; Z, Zephiran.

\begin{tabular}{|c|c|c|c|}
\hline Year & Mucolytic/decontaminant concentrations & Results & Reference \\
\hline 1963 & $4 \% \mathrm{NaOH}$ and $4 \% \mathrm{NaOH} / 20 \% \mathrm{NALC}$ & $\begin{array}{l}\mathrm{NaOH} / \mathrm{NALC} \text { yielded more positive cultures } \\
\text { than } \mathrm{NaOH} \text { alone }\end{array}$ & Kubica et al. (1963) \\
\hline 1966 & $\begin{array}{l}3 \% \mathrm{NaOH}, 4 \% \mathrm{NaOH}, 2 \% \mathrm{NaOH} / 20 \% \\
\text { NALC, Z/TSP and Z/20\% NALC }\end{array}$ & $\begin{array}{l}\mathrm{NaOH} / \mathrm{NALC} \text { was less lethal to mycobacteria than } \\
\text { the } 3 \text { and } 4 \% \mathrm{NaOH} \text {; Z/TSP did not have a significant } \\
\text { effect and } \mathrm{Z} / \mathrm{NALC} \text { had no loss in bacilli viability }\end{array}$ & $\begin{array}{l}\text { Krasnow \& Wayne } \\
(1966)\end{array}$ \\
\hline 1967 & $2 \% \mathrm{NaOH}$ and $2 \% \mathrm{NaOH} / 0.5 \% \mathrm{NALC}$ & $\begin{array}{l}\mathrm{NaOH} / \mathrm{NALC} \text { yielded more positive cultures than } \\
\mathrm{NaOH} \text { alone }\end{array}$ & $\begin{array}{l}\text { Lorian \& Lacases } \\
\text { (1967) }\end{array}$ \\
\hline 1969 & $\begin{array}{l}4 \% \mathrm{NaOH} / 20 \% \mathrm{NALC}, \mathrm{Z} / \mathrm{TSP} \text { and } \mathrm{Z} / 20 \% \\
\text { NALC }\end{array}$ & $\begin{array}{l}\text { Z/NALC had a higher contamination rate; } \mathrm{Z} / \mathrm{TSP} \text { and } \\
\mathrm{NaOH} / \mathrm{NALC} \text { yielded more positive cultures }\end{array}$ & $\begin{array}{l}\text { Krasnow \& Wayne } \\
\text { (1969) }\end{array}$ \\
\hline 1985 & $\begin{array}{l}4 \% \mathrm{NaOH}, 4 \% \mathrm{NaOH} / 0.5 \% \mathrm{NALC}, \mathrm{Z} / \mathrm{TSP} \\
\text { and } 5 \% \text { oxalic acid }\end{array}$ & $\begin{array}{l}\text { Oxalic acid was useful in sputum contaminated with } \\
\text { pseudomonas. Z/TSP and } \mathrm{NaOH} / \mathrm{NALC} \text { were not } \\
\text { as harsh to bacilli as } \mathrm{NaOH} \text { alone }\end{array}$ & $\begin{array}{l}\text { Kent \& Kubica } \\
(1985)\end{array}$ \\
\hline 1987 & $\begin{array}{l}3 \% \mathrm{NaOH} / 0.5 \% \mathrm{NALC} \text { and } 3.16 \% \mathrm{SDS} / 1 \% \\
\mathrm{NaOH}\end{array}$ & $\mathrm{NaOH} / \mathrm{NALC}$ had the highest contamination rate & $\begin{array}{l}\text { Salfinger \& Kafader } \\
\text { (1987) }\end{array}$ \\
\hline 1993 & $\begin{array}{l}2 \% \mathrm{NaOH} / 0.5 \% \mathrm{NALC} \text { and } 2 \% \mathrm{NaOH} / 0.5 \% \\
\mathrm{NALC} / 5 \% \text { oxalic acid }\end{array}$ & $\begin{array}{l}\text { Combined } \mathrm{NaOH} / \mathrm{NALC} / \text { oxalic acid reduced the } \\
\text { contamination rate to a greater extent than } \mathrm{NaOH} / \mathrm{NALC}\end{array}$ & $\begin{array}{l}\text { Whittier et al. } \\
\text { (1993) }\end{array}$ \\
\hline 2002 & $\begin{array}{l}2 \% \mathrm{NaOH} / 0.5 \% \mathrm{NALC} \text { and } 2 \% \mathrm{NaOH} / 0.5 \% \\
\mathrm{NALC} / 5 \% \text { oxalic acid (two-step method) }\end{array}$ & $\begin{array}{l}\mathrm{NaOH} / \mathrm{NALC} / \text { oxalic acid reduced contamination and } \\
\text { improved sensitivity by } 25 \%\end{array}$ & $\begin{array}{l}\text { Bange \& Böttger } \\
(2002)\end{array}$ \\
\hline 2006 & $\begin{array}{l}0.1 \% \text { Sputasol and } 1 \% \text { chlorhexidine digluconate } \\
\text { and } 2 \% \mathrm{NaOH} / 0.5 \% \mathrm{NALC} / 5 \% \text { oxalic acid }\end{array}$ & $\begin{array}{l}\text { Chlorhexidine yielded more positives than NaOH/NALC/ } \\
\text { oxalic acid but had a slight increase in contamination }\end{array}$ & Ferroni et al. (2006) \\
\hline
\end{tabular}

polymixin B (300 IU). A 0.5 standard McFarland suspension was prepared for each bacterial contaminant and swabbed onto MuellerHinton agar (Oxoid). The four antibiotic discs were placed onto the surface of the inoculated agar and incubated overnight at $37^{\circ} \mathrm{C}$ after which the results were read.

\section{RESULTS}

Bacterial contaminants were identified molecularly from contaminated LJ slopes and BacT/Alert liquid medium, as detailed in Table 2. With regard to the LJ slopes, the contaminants belonged to 11 different genera and were composed largely of Gram-negative organisms (45/53; $84.9 \%)$. There were two specimens that grew two different isolates, namely Escherichia coli/Enterococcus faecalis and $P$. aeruginosa/Serratia marcescens. The BacT/Alert contaminants belonged to 13 different genera, with $37 / 66(56.1 \%)$ of isolates being Gram-negative. There were two BacT/Alert bottles that had a mixed bacterial population, namely Staphylococcus epidermidis/Staphylococcus haemolyticus and P. aeruginosa/Staphylococcus epidermidis. With regard to the 11 paired LJ slopes/ BacT/Alert medium contaminants, seven contained P. aeruginosa, three contained Burkholderia cenocepacia and one contained Enterococcus faecalis. The same species of organisms were found concurrently in the BacT/Alert liquid medium and on the contaminated LJ slope.

The effects of the different selective decontamination protocols are shown in Table 3. Four of the six methods were effective at eliminating all culturable organisms present: $5 \%$ oxalic acid, $5 \%$ oxalic acid $/ 2 \% \mathrm{NaOH}, 5 \%$ oxalic acid $/ 4 \%$
$\mathrm{NaOH}$ and $1 \%$ chlorhexidine. The 2 and $4 \% \mathrm{NaOH}$ solutions were less effective as they were unable to eliminate all organisms, with the exception of $P$. aeruginosa.

The antibiotic susceptibility of all the bacterial contaminants isolated from the LJ slopes and BacT/Alert liquid medium to vancomycin, polymyxin $\mathrm{B}$, nalidixic acid and trimethoprim is shown in Table 4. Overall, 90/111 isolates $(81.1 \%)$ showed susceptibility to at least one antibiotic agent.

\section{DISCUSSION}

Breakthrough contamination of TB cultures is a problem in that it allows the overgrowth of another bacterium present in the sputum specimen, which can potentially mask the presence of M. tuberculosis. Fig. 1 shows the approximate doubling of breakthrough contamination rates that we found in our TB reference laboratory, increasing from $<5 \%$ to $\sim 10 \%$ between 2000 and 2005 . These baseline data $(\sim 4 \%)$ are in agreement with published breakthrough contamination rates in other parts of the world, including Timika in Indonesia and Adelaide in South Australia, where rates were $5.6 \%$ (Lumb et al., 2006). However, the approximate local doubling of this rate to $\sim 10 \%$ by 2005 gave rise to concerns as to why we were experiencing this, the ramifications for patient management/infection control that such a rise had on our ability to deliver an efficient $\mathrm{TB}$ service and how this contamination rate could successfully be reduced through method development/modifications. The reasons for the increase in breakthrough contamination in our reference laboratory remain unclear. We have 
Table 2. Description of identification and frequency of occurrence of bacterial contaminants isolated from $\mathrm{LJ}$ agar slopes and BacT/Alert liquid medium

\begin{tabular}{|c|c|c|}
\hline \multirow[t]{2}{*}{ Bacterial contaminant } & \multicolumn{2}{|c|}{ No. of contaminants } \\
\hline & $\begin{array}{c}\text { LJ slope } \\
\text { (\% of total } \\
\text { contaminants) }\end{array}$ & $\begin{array}{c}\text { BacT/Alert liquid } \\
\text { medium } \\
\text { (\% of total } \\
\text { contaminants })\end{array}$ \\
\hline Pseudomonas aeruginosa & $25(47.2 \%)$ & $20(30.3 \%)$ \\
\hline Serratia marcescens & $7(13.2 \%)$ & $2(3.0 \%)$ \\
\hline Escherichia coli & $4(7.5 \%)$ & \\
\hline Achromobacter sp. & & $1(1.5 \%)$ \\
\hline Burkholderia sp. & & $1(1.5 \%)$ \\
\hline Burkholderia cenocepacia & $3(5.7 \%)$ & $6(9.1 \%)$ \\
\hline Enterococcus faecalis & $3(5.7 \%)$ & $3(4.5 \%)$ \\
\hline Bacillus licheniformis & $1(1.9 \%)$ & \\
\hline Corynebacterium jeikeium & $1(1.9 \%)$ & \\
\hline Enterobacter sp. & $1(1.9 \%)$ & \\
\hline Klebsiella pneumoniae & $1(1.9 \%)$ & $1(1.5 \%)$ \\
\hline Lactobacillus rhamnosus & & $2(3.0 \%)$ \\
\hline Pseudomonas fluorescens & $1(1.9 \%)$ & \\
\hline Pseudomonas putida & $1(1.9 \%)$ & \\
\hline Proteus mirabilis & & $2(3.0 \%)$ \\
\hline Rahnella aquatilis & & $1(1.5 \%)$ \\
\hline Serratia sp. & $1(1.9 \%)$ & $1(1.5 \%)$ \\
\hline $\begin{array}{l}\text { Sphingobacterium } \\
\text { spiritivorum }\end{array}$ & & $1(1.5 \%)$ \\
\hline Staphylococcus sp. & & $3(4.5 \%)$ \\
\hline Staphylococcus aureus & $1(1.9 \%)$ & $3(4.5 \%)$ \\
\hline Staphylococcus epidermidis & $1(1.9 \%)$ & $15(22.7 \%)$ \\
\hline $\begin{array}{l}\text { Staphylococcus } \\
\text { haemolyticus }\end{array}$ & $1(1.9 \%)$ & $1(1.5 \%)$ \\
\hline $\begin{array}{l}\text { Stenotrophomonas } \\
\text { maltophilia }\end{array}$ & $1(1.9 \%)$ & $1(1.5 \%)$ \\
\hline Streptococcus sp. & & $1(1.5 \%)$ \\
\hline Streptococcus oralis & & $1(1.5 \%)$ \\
\hline Total & 53 & 66 \\
\hline
\end{tabular}

not altered our decontamination or selective methodologies during this period. One possibility is that the background commensal flora have become more resistant to the antibiotic selective agents that are contained within liquid TB culture medium, but we have not explored this systematically over time. Hence, it was the aim of the current study to identify reliably the bacterial agents responsible for the breakthrough contamination, and assess their ability to survive and persist after various chemical decontamination procedures, as well as to examine their susceptibility to antibiotics incorporated into liquid culture medium used in automated detection devices.

To date, few published studies have examined the bacteriological composition of breakthrough contaminants. In our study, we wished to have an accurate identification of such organisms; hence, we elected to identify all organisms using a 16S rRNA gene PCR and a sequenced-based identification method. Overall, we demonstrated a diverse range of bacterial organisms consisting of 17 genera, with P. aeruginosa and Staphylococcus epidermidis accounting for the most dominant Gram-negative and Gram-positive organisms isolated, respectively. The existence of breakthrough contamination is a combined failure of both the chemical decontamination stage and the selective component of the subsequent culture medium, whether in solid or liquid phase. Therefore, a re-examination of critical control points within the TB laboratory workup (Fig. 2) was logical in order to (i) challenge such breakthrough organisms to a variety of decontamination processes to rank previously published protocols for their ability to eliminate such contaminants, and (ii) examine the antibiotic susceptibility patterns of these organisms against the antibiotic agents found in liquid culture medium.

These contaminating bacteria can rapidly overgrow mycobacterial cultures, so specific decontamination methods are required. The most widely used decontamination method for Mycobacterium isolation from TB and other patients is the $N$-acetyl-L-cysteine (NALC)/NaOH decontamination

Table 3. Effect of six chemical decontaminants on the survival of the most frequently occurring breakthrough bacterial contaminants Results are shown as $\log _{10}$ c.f.u. $\mathrm{ml}^{-1}$. ND, No culturable organism detected.

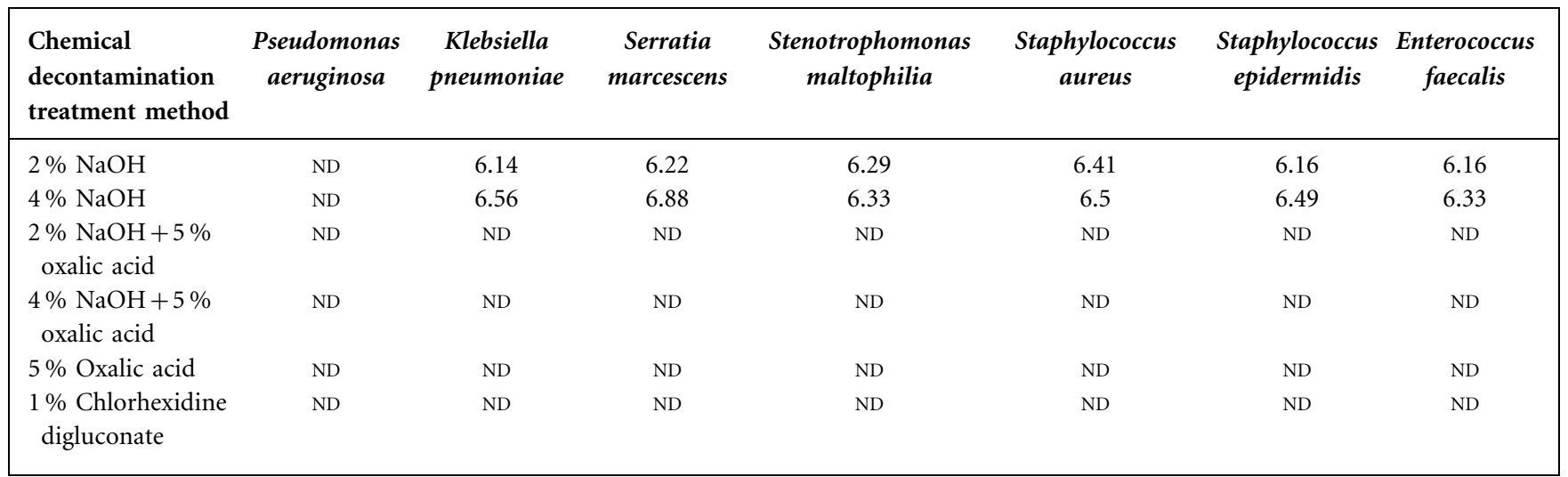


Table 4. Antibiotic susceptibility of 111 breakthrough contaminants to vancomycin, polymyxin B, nalidixic acid and trimethoprim

\begin{tabular}{|c|c|c|c|c|c|}
\hline Bacterial contaminant & No. isolates examined & Vancomycin & Polymyxin B & Nalidixic acid & Trimethoprim \\
\hline Serratia marcescens & 10 & 100 & 20 & 100 & 100 \\
\hline Escherichia coli & 3 & 100 & 0 & 100 & 100 \\
\hline Achromobacter sp. & 1 & 100 & 0 & 100 & 100 \\
\hline Burkholderia cenocepacia & 9 & 100 & 88.9 & 100 & 100 \\
\hline Enterococcus faecalis & 5 & 0 & 100 & 100 & 40 \\
\hline Bacillus licheniformis & 1 & 0 & 100 & 100 & 0 \\
\hline Corynebacterium jeikeium & 1 & 0 & 100 & 100 & 0 \\
\hline Enterobacter sp. & 1 & 100 & 0 & 100 & 0 \\
\hline Klebsiella pneumoniae & 2 & 100 & 100 & 100 & 100 \\
\hline Rahnella aquatilis & 1 & 100 & 0 & 0 & 100 \\
\hline Serratia sp. & 2 & 100 & 50 & 100 & 100 \\
\hline Sphingobacterium spiritivorum & 1 & 100 & 100 & 0 & 100 \\
\hline Staphylococcus sp. & 4 & 0 & 75 & 100 & 25 \\
\hline Staphylococcus aureus & 4 & 0 & 100 & 100 & 0 \\
\hline Staphylococcus epidermidis & 12 & 0 & 100 & 100 & 25 \\
\hline Staphylococcus haemolyticus & 1 & 0 & 100 & 100 & 0 \\
\hline Stenotrophomonas maltophilia & 2 & 100 & 50 & 100 & 50 \\
\hline Streptococcus sp. & 1 & 0 & 100 & 100 & 0 \\
\hline Streptococcus oralis & 1 & 0 & 100 & 100 & 0 \\
\hline
\end{tabular}

step; however, the two-step NALC/NaOH and oxalic acid method is a stronger decontamination method. These methods substantially decrease the contamination rate of sputum cultures and allow better recovery of mycobacteria than is possible by decontamination with $\mathrm{NALC} / \mathrm{NaOH}$ alone. However, the NALC/NaOH/oxalic acid method may affect the viability of mycobacteria (Ferroni et al., 2006) and consequently lead to false-negative results for samples with low mycobacterial loads. For many years, various scientists have compared different decontamination methods in order to discover the best method for sputum decontamination (Table 1). It can be seen from the table that $2 \% \mathrm{NaOH} / 0.5 \%$ NALC is the most common method used as it decreases the contamination rate and increases the number of positive cultures. An advantage of this method is the use of NALC, which provides good mucolytic action; however, a disadvantage is the short shelf-life of NALC. $\mathrm{NaOH}$ used alone is also effective and cheap; however, this method requires precise timing of the decontamination process to prevent any unnecessary killing of mycobacterial organisms. The most recent method used is the chlorhexidine method, which provided more positive cultures than the previous methods already discussed but did not alter the contamination rate greatly.

Overall, in the current study, we found that $\mathrm{NaOH}$ treatment alone, at a concentration of 2 or $4 \%$, was largely ineffective at eliminating the majority of the selection of common contaminants examined (Table 3 ). In addition, the presence of the four antibiotic agents in the liquid culture medium was ineffective at preventing breakthrough contamination in this medium. This is surprising in that the majority of isolates $(90 / 111 ; 81.1 \%)$ showed susceptibility to at least one antibiotic agent. Therefore, it would be expected that this sensitivity to at least one antibiotic in the liquid culture medium would have held and prevented breakthrough contamination from occurring. However, this was not the case, and breakthrough contamination was noted. This may have been the result of too low a concentration of antibiotic in the liquid culture medium compared with the concentration of antibiotic disc used in the susceptibility testing. Although it was outside the scope of the current study, future studies in this area should consider examining such susceptibility quantitatively using Etests to determine the MICs of antibiotics required to stop the growth of contaminants. Furthermore, it can be seen from Table 4 that nalidixic acid was relatively ineffective in preventing the growth of most of the contaminants tested. Any recommended increase in the concentration of antibiotic agents used in liquid culture medium to overcome increasing MIC values in contaminants needs to ensure ideally zero or minimal effect on the culturability of M. tuberculosis. This area therefore requires further evaluation, particularly by the manufacturers of commercially available liquid culture 


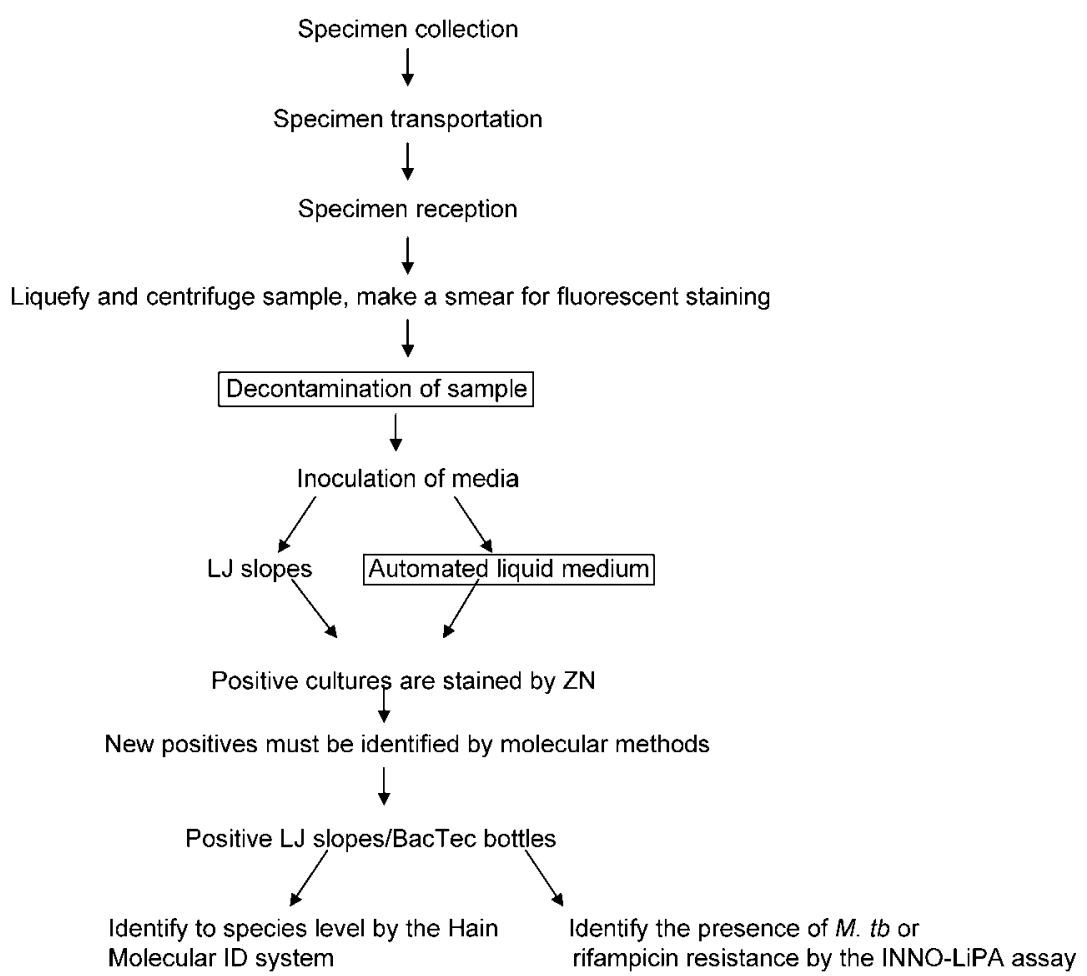

Fig. 2. Flow diagram detailing the stages of sputum processing for routine laboratory workup of TB. Boxed stages indicate the points that were investigated in the current study, as interventional critical control points to minimize breakthrough contamination. ZN, Ziehl-Neelsen stain; M. tb, M. tuberculosis. media, so that current media formulations are optimal at preventing breakthrough contamination but not so inhibitory that they begin to have a negative effect on recovery rates of $M$. tuberculosis.

One aspect of the current study that may need further exploration is that the decontamination processes were tested on bacteria in sterile PBS rather than on a sputum preparation. The medium that the bacteria are in will have a significant impact on the decontamination process; the chemical composition and consistency of sputum from, for example, a cystic fibrosis (CF) patient may vary considerably from that of a TB patient. We did consider this at the design stage of the experiment. We were aware of the differences in the rheology of sputum from different types of patients, such as sputum from CF patients versus sputum from TB patients. However, we were aware that any such studies on sputum, particularly from positive and potentially positive TB patients, would be problematic, as all such specimens would need to go to culture for service application, leaving no specimen for the current study. Therefore, we decided to examine potential decontamination protocols under standardized conditions, i.e. in PBS, as a starting position, because, if the downstream decontamination protocols were unable to kill contaminants within PBS, they would be unlikely to have any greater killing potential when the organisms could gain protection from entrapment in sputum. Further studies are now required to apply these optimal decontamination protocols in sterile PBS to the sputum type being examined, for example sputum from patients with TB, $\mathrm{CF}$ and non-CF bronchiectasis.
In conclusion, breakthrough contamination of TB cultures may be due to a diverse range of at least 17 different bacterial genera, with $P$. aeruginosa and Staphylococcus epidermidis accounting for the dominant contaminating flora. Employment of chemical decontaminating protocols solely involving $\mathrm{NaOH}$ may lead to higher rates of contamination. Where such contamination is encountered, TB laboratories should consider the reprocessing of such sputum with an alternative decontamination method, such as $1 \%$ chlorhexidine.

\section{ACKNOWLEDGEMENTS}

This work was financially supported through a Project Grant awarded by the Northern Ireland Chest, Heart and Stroke Association (NICHSA).

\section{REFERENCES}

Bange, F.-C. \& Böttger, E. C. (2002). Improved decontamination method for recovering mycobacteria from patients with cystic fibrosis. Eur J Clin Microbiol Infect Dis 21, 546-548.

Ferroni, A., Vu-Thien, H., Lanotte, P., Le Bourgeois, M., SermetGaudelus, I., Fauroux, B., Marchand, S., Varaigne, F., Berche, P. \& other authors (2006). Value of the chlorhexidine decontamination method for recovery of nontuberculous mycobacteria from sputum samples of patients with cystic fibrosis. J Clin Microbiol 44, 22372239.

Kent, P. T. \& Kubica, P. G. (1985). Public Health Bacteriology, a Guide to the Level III Laboratory. Atlanta, GA: Centers for Disease Control and Prevention. 
Krasnow, I. \& Wayne, L. G. (1966). Sputum digestion. I. The mortality rate of tubercle bacilli in various digestion systems. Tech Bull Regist Med Technol 36, 34-37.

Krasnow, I. \& Wayne, L. G. (1969). Comparison of methods for tuberculosis bacteriology. Appl Microbiol 18, 915-917.

Kubica, G. P., Dye, W. E., Cohn, M. L. \& Middlebrook, G. (1963). Sputum digestion and decontamination with $\mathrm{N}$-acetyl-L-cysteine-sodium hydroxide for culture of mycobacteria. Am Rev Respir Dis 87, 775-779.

Lorian, V. \& Lacases, M. L. (1967). N-Acetyl-L-cysteine sputum homogenization and its mechanism of action on isolation of tubercle bacilli. Dis Chest 51, 275-277.

Lumb, R., Ardian, M., Waramori, G., Syahrial, H., Tjitra, E., Maguire, G. P., Anstey, N. M. \& Kelly, P. M. (2006). An alternative method for sputum storage and transport for Mycobacterium tuberculosis drug resistance surveys. Int J Tuberc Lung Dis 10, 172-177.

Salfinger, M. \& Kafader, F. M. (1987). Comparison of two pretreatment methods for detection of mycobacteria of BACTEC and Lowenstein-Jensen slants. J Microbiol Methods 6, 315-321.

Whittier, S., Hopfer, R. L., Knowles, M. R. \& Gilligan, P. H. (1993). Improved recovery of mycobacteria from respiratory secretions of patients with cystic fibrosis. J Clin Microbiol 31, 861864.

Xu, J., Stanley, T., Millar, B. C., McClurg, R. B., Shaw, A., Crothers, L., Goldsmith, C. E., Rooney, R. J., Loughrey, A. \& other authors (2008). Difficult-to-identify bacteria: how use of $16 \mathrm{~S}$ rDNA PCR and gene sequencing can help. Br J Biomed Sci 65, 33-36. 